\title{
The effects of a counter-current interstitial flow on a discharging hourglass
}

\author{
B. K. Muite, M. L. Hunt, ${ }^{\text {a) }}$ and G. G. Joseph \\ Division of Engineering and Applied Science, California Institute of Technology, MS 104-44, Pasadena, \\ California 91125
}

(Received 22 September 2003; accepted 17 June 2004; published online 2 August 2004)

\begin{abstract}
This work experimentally investigates the effects of an interstitial fluid on the discharge of granular material within an hourglass. The experiments include observations of the flow patterns, measurements of the discharge rates, and pressure variations for a range of different fluid viscosities, particle densities and diameters, and hourglass geometries. The results are classified into three regimes: (i) granular flows with negligible interstitial fluid effects; (ii) flows affected by the presence of the interstitial fluid; and (iii) a no-flow region in which particles arch across the orifice and do not discharge. Within the fluid-affected region, the flows were visually classified as lubricated and air-coupled flows, oscillatory flows, channeling flows in which the flow preferentially rises along the sidewalls, and fluidized flows in which the upward flow suspends the particles. The discharge rates depends on the Archimedes number, the ratio of the effective hopper diameter to the particle diameter, and hourglass geometry. The hopper-discharge experiments, as well as experiments found in the literature, demonstrate that the presence of the interstitial fluid is important when the nondimensional ratio $(N)$ of the single-particle terminal velocity to the hopper discharge velocity is less than 10. Flow ceased in all experiments in which the particle diameter was greater than $25 \%$ of the effective hopper diameter regardless of the interstitial fluid. (C) 2004 American Institute of Physics. [DOI: 10.1063/1.1781158]
\end{abstract}

\section{INTRODUCTION}

For centuries, the hourglass has been used as a method to measure the passage of time. The time-keeping principles are simple: the volume of material that has discharged from the upper bulb or hopper is linearly proportional to the time that has passed. Hence the flow rate through the hopper is constant with time and independent of the amount of material above the orifice. Because the discharge rate is independent of the height of the bed, the principles governing the flow rate differ significantly from flow of a Newtonian fluid. As a result, the study of flow in an hourglass is useful in understanding the principal mechanisms governing flows of granular materials.

Most hourglasses are filled with sand and air, although some use fine glass beads or ground eggshells. As the material falls from the upper hopper, air moves upward through the permeable particle bed resulting in a countercurrent coupling of the airflow and the falling material. Consequently, the discharge rate in an hourglass may depend on the upward flow of interstitial air. The "world's largest hourglass," located at the Nima Sand Museum in Shimane Prefecture, Japan, is $5.2 \mathrm{~m}$ in height, $1 \mathrm{~m}$ in diameter and contains $\sim 1$ ton of sieved sand ( $0.13 \mathrm{~mm}$ diameter). The hourglass is flipped at midnight on New Year's eve. To maintain a constant flow rate $(0.032 \mathrm{~g} / \mathrm{s})$ through the orifice $(0.13 \mathrm{~mm}$ diameter $)$, the air pressure within the hourglass is controlled to compensate for the variable temperature conditions throughout the year. Hence, the countercurrent airflow can control the passage of the sand, and in this case, control the measurement of time.

a)Electronic mail: hunt@caltech.edu
In an hourglass containing relatively large particles, the discharge rate may be unaffected by the interstitial gas. For these conditions, the flow of the material is similar to that found in an open ventilated hopper. An understanding of flow in a discharging hopper has developed from numerous analytical and experimental studies as described by Nedderman. ${ }^{1}$ Figure 1 shows a sketch of a cylindrical hourglass and includes the half angle of the cone-shaped hopper. Particles enter the hopper from an upper bin in which the material moves as a plug. As the material moves towards the hopper, the material undergoes strain because of the change in geometry. If the granular material is close to the random close packing limit (a solid fraction near 0.63) then the material must dilate for shear to occur. If the granular material is poured into the hopper so that it is close to the random loose packing limit (a solid fraction near 0.55), the shearing may occur without much further dilation. Typically, the granular bed will settle under the influence of gravity and small disturbances; hence for most flows the volume fraction will be less than the random loose packing limit. In moving through the hopper, the granular material becomes easier to shear, reaching a location referred to as the arch at which compressive stresses are not transmitted through the granular material. Above the arch, the walls support most of the weight of the particles in the hopper. Once the grains pass the arch, they jostle past each other towards the orifice. The gravity driven jostling processes below the arch and above the orifice determine the discharge rate of a dry hopper.

As outlined in the text by Nedderman, ${ }^{1}$ when interstitial fluid effects are insignificant the discharge rate of a particular monodisperse granular material depends primarily on gravity and the size of the hopper orifice. Hence, the average dis- 


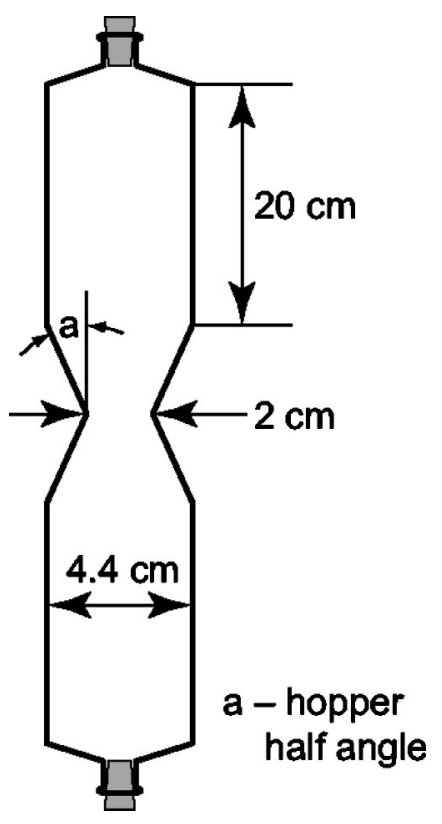

FIG. 1. Diagram of $15^{\circ}$ and $30^{\circ}$ cylindrical hourglasses.

charge velocity of the particles $w_{s}$ can be scaled by the characteristic hopper discharge rate $w_{o}$,

$$
W=w_{s} / w_{o}=w_{s} / C\left[g\left(1-\rho_{f} / \rho_{s}\right) D^{\prime}\right]^{1 / 2},
$$

where $w_{o}=C\left[g\left(1-\rho_{f} / \rho_{s}\right) D^{\prime}\right]^{1 / 2}$, which includes the effects of particle buoyancy on gravitational acceleration $g$ and $D^{\prime}$ is the effective orifice diameter. This diameter $D^{\prime}=D-k d$ is defined using the Beverloo correction, ${ }^{1}$ where $D$ is the orifice diameter for a cylindrical hopper and $d$ is the particle diameter. The Beverloo correction $k$ has been empirically introduced to account for the reduction in orifice size because the center of a particle must be at least $d / 2$ from the edge of the hopper; the value is slightly larger than unity. For planar hopper of depth $l$ and an orifice width $b$ the rate determining effective orifice width is the hydraulic diameter $D_{h}=4 A / P$, which is defined from the perimeter $P$ and the cross-sectional area $A$ of the region

$$
D_{h}=\frac{4(b-k d)(l-k d)}{2(l+b-2 k d)} .
$$

In Eq. (1), the adjustable constant $C$ depends on the friction angle of the particles and the hopper angle and is usually determined by experiment; however, the value is typically close to unity. Here, the value of $C$ is chosen as unity in scaling the characteristic velocity $w_{o}$.

Several recent studies ${ }^{2-4}$ have examined unsteady flow or "ticking" in an hourglass. Wu et al. ${ }^{2}$ reported that the flow out of a cylindrical hourglass appeared to be intermittent with active (flow) and inactive (no flow) phases. Their measurements presented the mass of the discharging material over time and demonstrated that the intermittent behavior was due to the upward flow of air. During the active phase, the pressure in the upper bulb drops until the pressure difference with respect to the atmosphere reaches a maximum; at this point the air/particle interface is stabilized and the flow of grains stops. In the passive phase, air seeps through the stationary bed resulting in a pressure increase in the upper bulb. The pressure in the bulb reaches a value at which the pressure difference can no longer support the particles, and the flow resumes. This intermittent behavior occurred for particle diameters in the range from 40 to $115 \mu \mathrm{m}$ using an orifice with diameter of $\sim 2 \mathrm{~mm}\left(16>D^{\prime} / d>49\right)$. For particles larger than $115 \mu \mathrm{m}\left(16<D^{\prime} / d\right)$, the material appeared to discharge continuously until reaching a limit when the particles were large enough to arch across the orifice and stop the flow; for particles smaller than $40 \mu \mathrm{m}\left(D^{\prime} / d<49\right)$ interparticle forces prevented the material from discharging. A later study in the same laboratory ${ }^{3}$ found a low-density bubble region within the neck of the hourglass during the active phase of the discharge cycle. The bubble would oscillate and move upward into the packed material during the inactive phase.

A recent study by Veje and Dimon ${ }^{4}$ also focused on the dynamics of intermittent flow in the closed upper chamber of an hourglass with a gradually varying stem. The experimental measurements indicate that for sufficiently small particles $\left(\sim 100 \mu \mathrm{m}\right.$ glass spheres; $\left.D^{\prime} / d=29\right)$ the air/particle interface is stationary in position, but breaks during regular intervals allowing the material to exit the hopper. For slightly larger particles $\left(\sim 200 \mu \mathrm{m}\right.$ glass spheres; $\left.D^{\prime} / d=14\right)$ the interface oscillates.

Comparable studies on hoppers suggest that flow rates in an unventilated hopper are reduced when compared to ventilated flow if the particles are sufficiently small. ${ }^{5}$ If the interstitial fluid is a liquid, the countercurrent liquid flow may have a pronounced effect on the discharge rate and discharge pattern; for example, Ducker et al. ${ }^{5}$ have suggested that the return flow fluidized the granular material.

Although liquid-filled hourglasses are not important for time keeping, similar flow phenomena are found in industries that handle granular materials, slurries, or debris flows. The current research uses a simple hourglass geometry to examine the effect of fluid properties on the discharge rate and discharge pattern of different interstitial fluids and granular materials. The important physical properties in determining hourglass discharge rate are gravity, particle density, fluid density, particle diameter, fluid dynamic viscosity, orifice diameter, and hourglass geometry. The surface roughness and polydispersity of the particles may also be important, ${ }^{4}$ but were not explicitly examined in this study.

\section{EXPERIMENTAL METHOD}

To examine interstitial fluid effects, several different hourglass shaped vessels of varying complexity were made as shown in Figs. 1 and 2. Two hourglasses were made from blown glass with $15^{\circ}$ and $30^{\circ}$ hopper half angles; a third was machined out of acrylic with a $90^{\circ}$ hopper half angle. The three vessels were cylindrical with an orifice of $2 \mathrm{~cm}$; the region of constant cross-sectional area $(4.4 \mathrm{~cm}$ in diameter for the $15^{\circ}$ and $30^{\circ}$ hourglasses; $5 \mathrm{~cm}$ for the $90^{\circ}$ hourglass) was at least $20 \mathrm{~cm}$ in length. To remove air bubbles in the glass devices, a cylinder was fitted over the top hole and a leak-proof seal made between it and the hourglass. The cylinder was filled with the same fluid so that the vessel could 


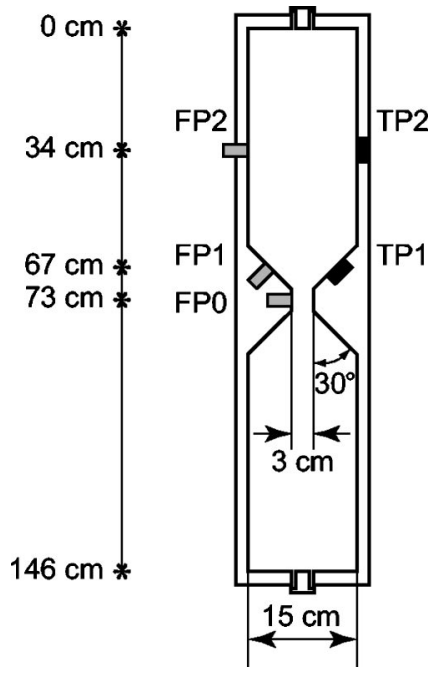

FIG. 2. Diagram of the two-dimensional hourglass. The locations of total pressure (TP) and fluid pressure (FP) transducers are shown in the upper bin.

be capped while immersed. For the experiments, the cylinder was then removed after capping. The acrylic vessel had a bleed screw through which fluid could be injected to remove unwanted air.

The fourth apparatus (Fig. 2) was a two-dimensional (2D) channel that was made by sandwiching a steel lined aluminum frame between front and back acrylic windows. This apparatus was $1.5 \mathrm{~m}$ tall, $15 \mathrm{~cm}$ wide, and $4.4 \mathrm{~cm}$ deep, with a $30^{\circ}$ half angle and a $3 \mathrm{~cm}$ orifice width; it was supported on a swivel stand. The vessel contained three fluid gauge pressure transducers (Druck® PMP 1225) that could measure pressures between 0 to $35 \mathrm{kPa}$ to a resolution of $0.1 \mathrm{kPa}$. These transducers were located behind flush mounted porous screens to prevent particles from contacting the sensing area without altering flow conditions. The hourglass also contained two flush mounted total pressure transducers (Honeywell $\left.{ }^{\circledR} \mathrm{AB} / \mathrm{HP}\right)$ that measured pressure due to fluid and particles in contact with a flat $1.9 \mathrm{~cm}$ diameter sensing area. These transducers measured pressures between 0 and $40 \mathrm{kPa}$ to a resolution of $0.2 \mathrm{kPa}$ and were calibrated during filling by measuring the hydrostatic head of the fluid above each transducer. Transducer locations are illustrated in Fig. 2.

During discharge of the cylindrical and 2D hourglasses, the location of the fluid/particle interface in the bin was recorded on video and digitized. This height was found from the images by locating five points across the bin that had the sharpest change in intensity; in the experiments the difference in height along this line was less than $0.5 \mathrm{~cm}$. A horizontal line was fit to these points to find the average interface height and the average bin discharge velocity. The average particle velocity at the orifice was calculated using the bin discharge velocity and the effective areas (based on the Beverloo correction) of the orifice and bin sections. The interface was tracked over a 7-10 cm distance in the cylindrical hourglasses and over at least a $25 \mathrm{~cm}$ distance in the 2D hourglass. To reduce end effects, tracking started once a clear interface formed and stopped at the top of the hopper section.
Experiments were repeated at least seven times for each data set.

In the 2D facility, a computer data acquisition system collected transducer output at frequencies from $2 \mathrm{~Hz}$ up to $40 \mathrm{~Hz}$. Video and pressure recordings were synchronized by a strobe light that was triggered with transducer data acquisition. To reduce hysteretic errors, transducer zeros were recalibrated after each run by checking transducer outputs after discharge and calculating the appropriate hydrostatic pressure.

Glass beads and steel shot were discharged in air, pure water, or in water-glycerin mixtures, while lead shot was discharged in water or silicon oil. The steel-shot water experiments also contained $0.1 \%$ sodium nitrite to retard corrosion. The glass beads were of various diameters $(0.6,1.6$, $3.0,4.0$, and $6.0 \mathrm{~mm}$ ) with a density of $2.5 \mathrm{~g} / \mathrm{cm}^{3}$; the lead shot $(0.47,2.0$, and $3.5 \mathrm{~mm}$ diameter $)$ has a density of $11.0 \mathrm{~g} / \mathrm{cm}^{3}$; the steel shot were $1.4 \mathrm{~mm}$ diameter and a density of $7.9 \mathrm{~g} / \mathrm{cm}^{3}$. The metal and $1.6 \mathrm{~mm}$ glass particles had rougher surfaces and were less spherical than the other glass particles. The fluids included air (viscosity $0.018 \mathrm{cP}$; density of $0.0012 \mathrm{~g} / \mathrm{cm}^{3}$ ), silicon oil (viscosity of 100,500 , or $1000 \mathrm{cP}$; density of $1 \mathrm{~g} / \mathrm{cm}^{3}$ ) and water-glycerin mixtures (viscosity from 1 to $600 \mathrm{cP}$; density from 0.995 to $1.2 \mathrm{~g} / \mathrm{cm}^{3}$ ). The density and viscosity of the water-glycerin mixtures were calculated from the temperature and specific gravity measurements of these mixtures.

\section{RESULTS}

\section{A. Flow patterns and discharge rates}

Several different flow patterns were observed and visually classified as dry, lubricated, oscillatory, channeling, or fluidized. In a dry flow, air was the interstitial fluid. The lubricated flows appeared visually similar to dry flows but the discharge rate was decreased due to the presence of the viscous liquid.

In an oscillatory flow, fluid bubbles appeared in the throat of the hourglass as shown in the series of images in Fig. 3 taken at $1 \mathrm{~s}$ intervals. In the first image the bubble appears at the lower edge of the orifice. Approximately $1 \mathrm{~s}$ later, the bubble has broken apart and the exiting stream is directed to the left. The higher velocity fluid moves into the hopper region as shown in the third image and begins to diminish as it moves further up into the hopper as seen in the fourth image. Subsequent figures show the stream migrating to the right with the appearance of a bubble.

In channeling flow, the bed showed a distinct region of preferential upward fluid flow near the hourglass wall as observed in Fig. 4. In the figure, the upwardly moving fluid dragged the blurred particles. The channel extended from the orifice to the upper surface of the bed and was formed on the side of the hourglass that faced upwards when the hourglass was inverted.

Under certain conditions, the upward flow fluidized sections of the bed. For some flows such as the $0.6 \mathrm{~mm}$ glass beads in water, the fluidized section appeared unsteady with disturbances propagating through the bed. However, these disturbances were not observed for slightly larger particles in 


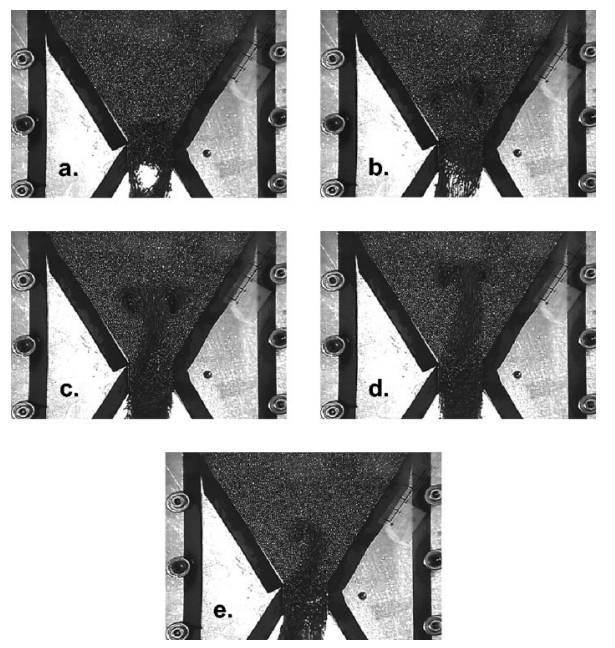

FIG. 3. Photographs showing the propagation of a fluid bubble for oscillating flow of $1.4 \mathrm{~mm}$ steel shot in water. (a) Slow flow, beads packed near orifice. (b) Fluid bubble begins to move up into hopper. (c) Fluid bubble continues moving up into hopper. (d) Fluid bubble starts to contract. (e) Slow flow returns.

more viscous fluids. For other flows $(1.6$ and $3.0 \mathrm{~mm}$ in water-glycerin mixtures), fluidization was apparent only near the orifice section of the hourglass.

Measurements were made of the discharge rates for the flow regimes. Figure 5 shows examples of the interface height versus time in the 2D hourglass for $6 \mathrm{~mm}$ glass beads in air, $4 \mathrm{~mm}$ glass beads in water (lubricated), $1.4 \mathrm{~mm}$ steel shot in water (oscillatory), $1.4 \mathrm{~mm}$ steel in a $20 \mathrm{cP}$ waterglycerin mixture (fluidized) and $3 \mathrm{~mm}$ glass beads in a $96 \mathrm{cP}$ water-glycerin solution (channeling). For the dry flow, the initial interface height was smaller because a significant amount of granular material discharged during flipping of the hourglass. For all of the curves, the discharge rate appears to be relatively constant with small fluctuations. Note that the discharge rate of the steel particles in water is approximately twice as fast as for glass beads in water.

Figure 6 presents the dimensionless discharge speed $W$ as a function of $d / D^{\prime}$ for glass beads in air or in water. For simplicity and as a best fit of the data, the value of the Beverloo constant, $k$, was taken as 1.1 for all of the experiments and geometries. If the parameter $k$ were taken as 0 for discharge in air, the data for the 2D hopper would show a decrease in the discharge rate with increasing particle size.

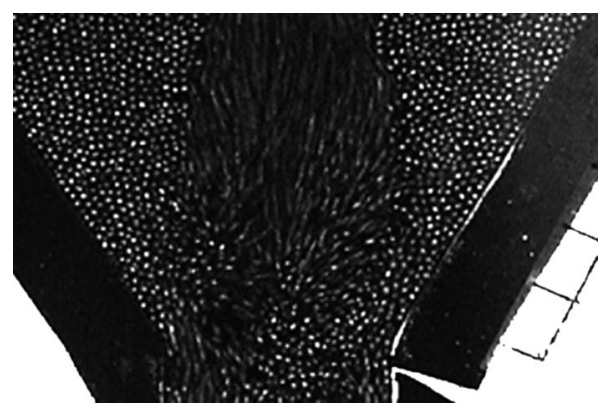

FIG. 4. Close-up of hopper in channeling discharge of $1.4 \mathrm{~mm}$ steel shot in a $20 \mathrm{cp}$ water-glycerin mixture.

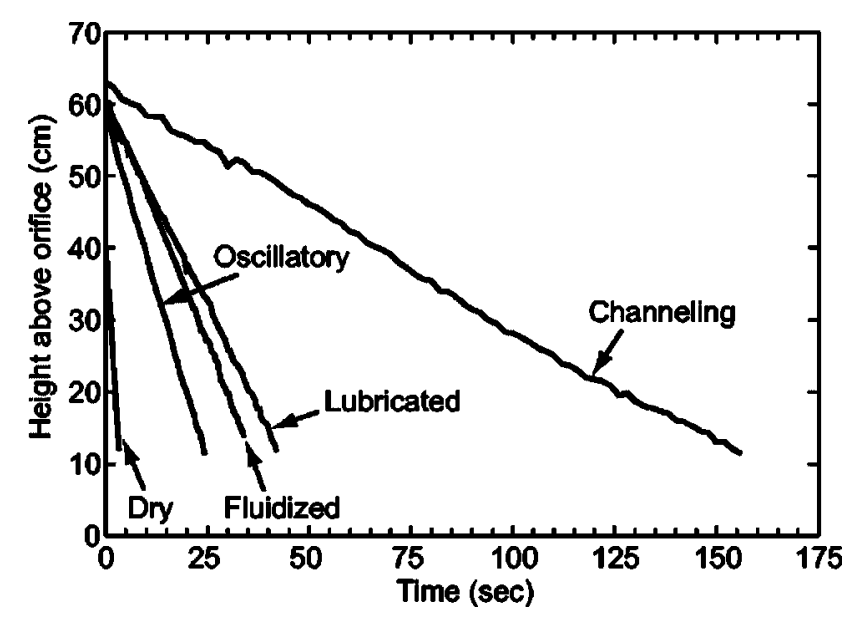

FIG. 5. Interface height vs time in the 2D hourglass; dry corresponds to $6 \mathrm{~mm}$ glass beads in air; lubricated $4 \mathrm{~mm}$ glass beads in water; oscillatory $1.4 \mathrm{~mm}$ steel shot in water; fluidized $1.4 \mathrm{~mm}$ steel shot in $20 \mathrm{cP}$ waterglycerin mixture; channeling $3 \mathrm{~mm}$ glass beads in $96 \mathrm{cP}$ water-glycerin mixture.

For dry flows and the largest values of $d / D^{\prime}$, the values of $W$ are close to unity; the variations reflect the differences in the hopper geometries and the constant $C$. Interestingly, the two experiments for the $0.6 \mathrm{~mm}$ glass spheres in the cylindrical vessels show a drop in discharge rate of almost $50 \%$ as compared with the values for the larger diameter spheres, suggesting that discharge rate is reduced by the upward flow of air. This drop is not found for the 2D geometry, because the smallest value of $d / D^{\prime}$ is for $0.47 \mathrm{~mm}$ lead shot, which is denser than glass and less affected by the interstitial fluid. The error bars show the standard deviation calculated from $\sim 10$ measurements. For the dry flows the standard deviation was typically $3 \%-4 \%$ of the average value.

Figure 6 also includes the discharge with water for several different particle diameters. In all of these flows, the discharge was described as lubricated. Compared with the dry flows, the discharge rate decreased by approximately a factor of 8 and showed a rather weak dependence on particle diameter. The water discharge rates also appeared to be largely independent of the device geometry. The standard

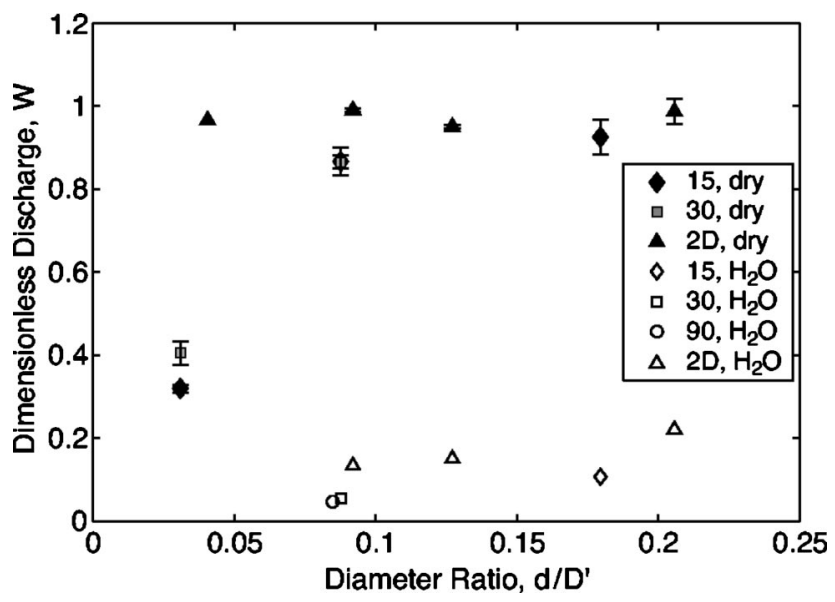

FIG. 6. Dimensionless discharge rate $W$ as a function of $d / D^{\prime}$ for glass beads in air and in lubricated flows in water. 


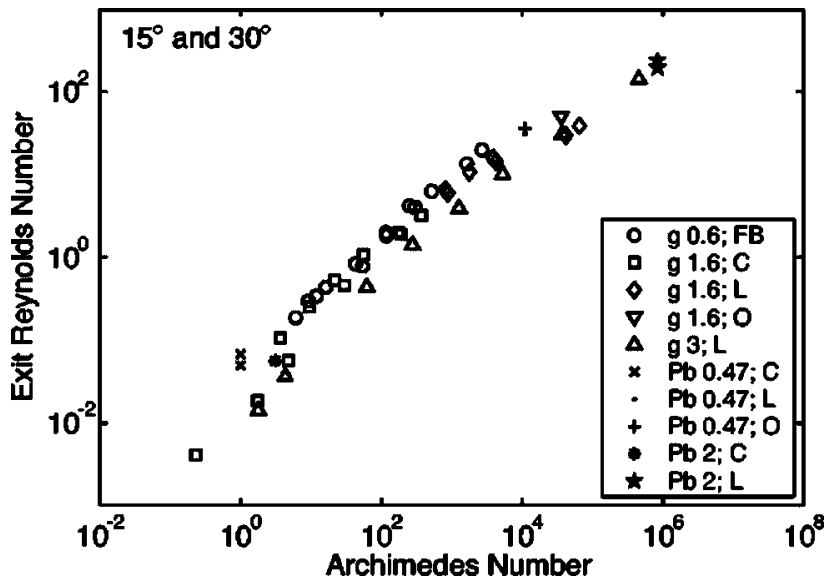

FIG. 7. Reynolds number based on the particle velocity at the exit of the hopper as a function of Archimedes number for flows in the $15^{\circ}$ and $30^{\circ}$ hourglasses for glass $(\mathrm{g})$ and lead $(\mathrm{Pb})$ particles of different diameters (in $\mathrm{mm})$. The data do not include dry flows. The labeling indicates lubricated $(\mathrm{L})$, oscillatory $(\mathrm{O})$, fluidized bed $(\mathrm{FB})$, and channeling $(\mathrm{C})$ behaviors.

deviation of these measurements was at most 7\%; the error bars are not shown because they were of the same size of the data symbols in the linear scale.

Although the flows demonstrated a varying dependence on fluid and solid properties, the discharge rates can be explained relatively simply. Consider the discharge of large dense particles from a hopper in air. As the material moves downwards in the hopper, the material dilates and accelerates to the hopper exit velocity. Upon exiting the orifice, the assembly of particles continues to accelerate from the exit velocity to a terminal or free fall velocity. However, as the particle size is decreased or if the surrounding fluid is a liquid, the hopper velocity may begin to approach the terminal velocity. Under these conditions, the terminal velocity would then act to control the discharge rate rather than the rate governed by the size of the hopper opening.

Figure 7 shows a plot for the $15^{\circ}$ and the $30^{\circ}$ hoppers of the particle Reynolds number using the velocity of a particle as it exits the hopper, $\operatorname{Re}=\rho_{f} w_{s} d / \mu$, where $\mu$ is the fluid viscosity as a function of the Archimedes number, $\mathrm{Ar}$ $=\rho_{f}\left(\rho_{s}-\rho_{f}\right) g d^{3} / \mu^{2}$, a parameter found in the particle settling literature. Note, for a single-particle settling at the Stokes terminal velocity, Re equals $\mathrm{Ar} / 18$ using the Stokes velocity $\left[d^{2} g\left(\rho_{s}-\rho_{f}\right) / 18 \mu\right]$ as the characteristic speed. The figure shows that velocity increases with Ar and suggests a secondary dependence on particle size. This variation appears to be linked to the exhibited flow regime. The figure also includes data points for lead shot $(2 \mathrm{~mm}$ and $0.47 \mathrm{~mm})$ in water and in either a $100 \mathrm{cP}(0.47 \mathrm{~mm}$ particles $)$ or a $500 \mathrm{cP}(2 \mathrm{~mm})$ silicon oil. The lead data follows the data for the glass spheres with the exception of the $0.47 \mathrm{~mm}$ particles in $100 \mathrm{cP}$ fluid where the exit Reynolds number is somewhat larger than would be expected. Experiments were also performed with the $0.47 \mathrm{~mm}$ lead in the $500 \mathrm{cP}$ fluid. For this combination of parameters, the bed remained suspended after inverting the hopper, making it impossible to define a clear interface between the receding granular media and the suspending fluid.

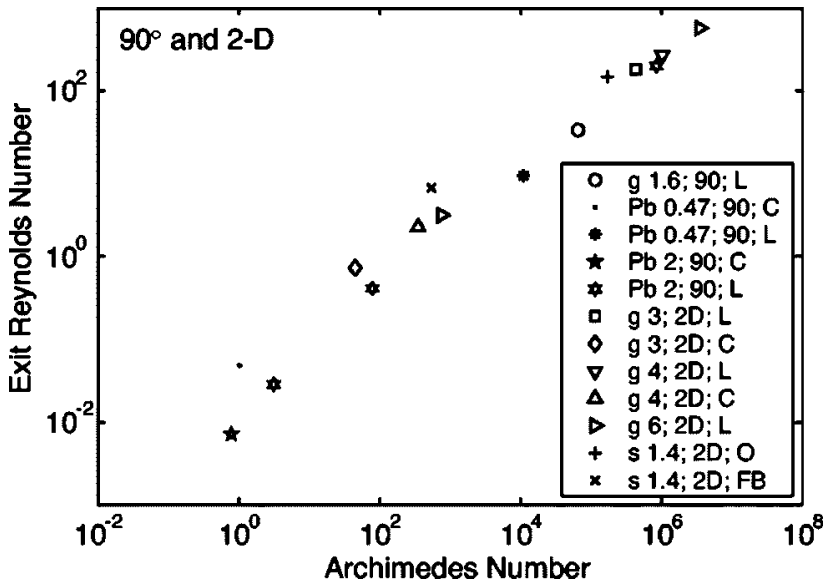

FIG. 8. Reynolds number based on the particle velocity at the exit of the hopper as a function of Archimedes number for flows in the $90^{\circ}$ and $2 \mathrm{D}$ hourglasses for glass $(\mathrm{g})$, steel $(\mathrm{s})$, and lead $(\mathrm{Pb})$ particles of different diameters (in $\mathrm{mm}$ ). The labeling indicates lubricated (L), oscillatory (O), fluidized bed $(\mathrm{FB})$, and channeling $(\mathrm{C})$ behaviors.

Figure 8 shows the exit Reynolds number increases with increasing Archimedes number for the $90^{\circ}$ hoppers and for the $2 \mathrm{D}$ system. These hourglasses also show similar trends regarding the types of flow. In Figs. 7 and 8, the standard deviation in the measured exit Reynolds number was also calculated. For most of the measurements, the standard deviation was less than $10 \%$ of the mean of the exit Reynolds number.

Figure 9 is a map of the flows in terms of Archimedes number and $d / D^{\prime}$ for all of the experiments with a liquid interstitial fluid. Lubricated flows were found for the largest Archimedes numbers and the largest values of $d / D^{\prime}$. Oscillatory flow was found in the $15^{\circ}$ and $2 \mathrm{D}$ geometries for $\mathrm{Ar}$ $\approx 10^{4}$; for the same Ar, the flow was described as lubricated in $30^{\circ}$ and $90^{\circ}$ geometries. The fluidized bed data was primarily found in the smallest values of $d / D^{\prime}$ for $10^{1}<\mathrm{Ar}<10^{4}$. In the $15^{\circ}$ and $30^{\circ}$ geometries for larger values of $d / D^{\prime}$, the material in the hopper was fluidized; however, the material above the hopper remained in contact and

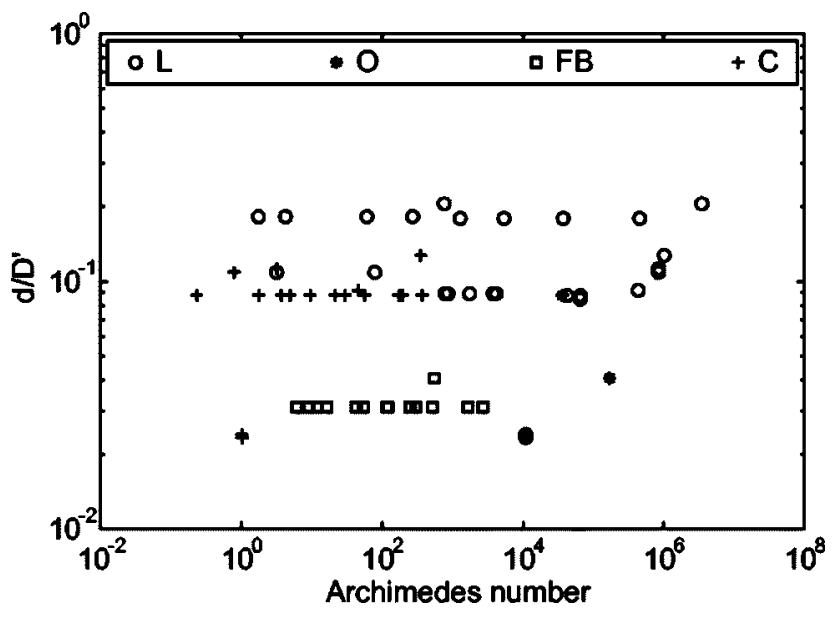

FIG. 9. Regime map for flows with an interstitial liquid. The labeling indicates lubricated $(\mathrm{L})$, oscillatory $(\mathrm{O})$, fluidized bed $(\mathrm{FB})$, and channeling $(\mathrm{C})$ behaviors. 


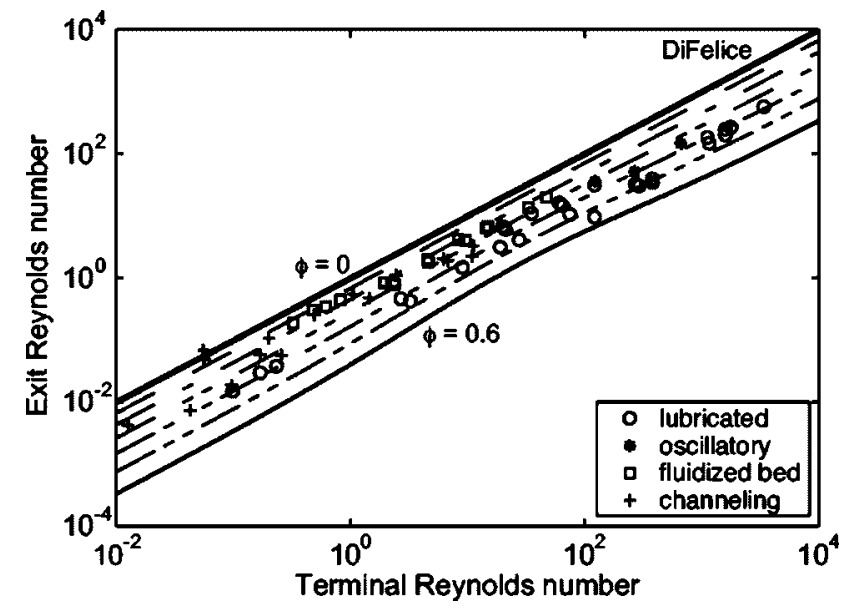

FIG. 10. Reynolds number based on the particle velocity at the exit of the hopper as a function of terminal Reynolds number for a single particle. The lines correspond to solid fraction from 0 to 0.6 based on a DiFelice's modified Richardson-Zaki model.

in the lubricated state, and thus described as lubricated. The lubricated data in the $90^{\circ}$ geometry differed from the other data, which may be associated with the stagnant region along the sidewalls and above the orifice. The channeling data occurred for $d / D^{\prime} \approx 0.1$ and $10^{-1}<\mathrm{Ar}<10^{3}$ and for smaller values of $d / D^{\prime}$ for $\operatorname{Ar}<1$.

For a single-particle falling in a quiescent fluid, the terminal Reynolds number $\left(\operatorname{Re}_{t}=\rho_{f} d w_{t} / \mu\right.$ with $w_{t}$ as the terminal velocity for a single particle) can be defined from a balance of gravitational and buoyancy forces to drag forces, and is calculated from the following equation:

$$
C_{d} \mathrm{Re}_{t}^{2}=\frac{4}{3} \mathrm{Ar}
$$

in which the analytical form of the drag coefficient $C_{d}$ depends nonlinearly on the Reynolds number. Empirical relations between the drag coefficient and the Reynolds number can be found in the literature, such as the relations suggested by Clift, Grace and Weber; ${ }^{6}$ note for Stokes flow, $C_{d}$ $=24 / \operatorname{Re}_{t}$. Figure 10 shows the results of the exit Reynolds number as a function of the terminal Reynolds number for all of the experiments (except the dry data). The results show that the exit Reynolds number is less than the value of the terminal Reynolds number for a single particle with the exception of the $0.47 \mathrm{~mm}$ lead particles in the $100 \mathrm{cP}$ fluid in which the discharge Reynolds number is approximately equal to the terminal Reynolds number. This exception results from circulation currents set up by the channeling. Particle settling speeds have been observed that are faster than the terminal velocity in enhanced sedimentation studies. ${ }^{7}$

The settling velocity of a bed of particles can be related to the terminal velocity of a single particle and the bed solid fraction using the classic Richardson-Zaki ${ }^{8}$ correlation with modifications as presented by DiFelice. ${ }^{9}$ The lines shown in Fig. 10 indicate the calculated settling Reynolds number for solid fraction from 0 to 0.6 as a function of the singleparticle terminal Reynolds number. ${ }^{9}$ For the lubricated flows at the highest values of $\operatorname{Re}_{t}$ (corresponding to the largest values of $\mathrm{Ar}$ ), the experimental data correspond closest to the line for a solid fraction between $\phi=0.4$ and $\phi=0.5$. The

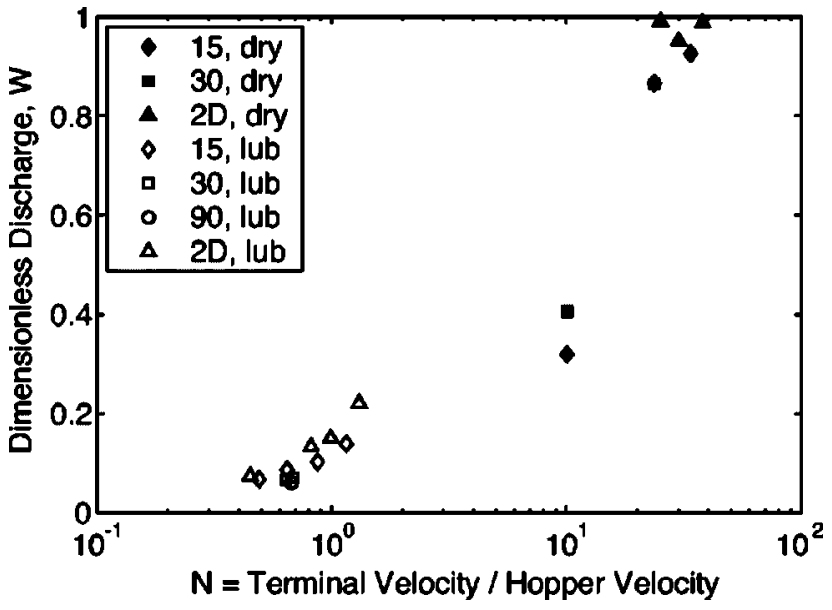

FIG. 11. Dimensionless discharge rate $W$ as a function of $N$ for dry and lubricated flows.

oscillatory data fall near the line for $\phi=0.4$, while the channeling and fluidized bed flows correspond to somewhat lower values. These solid fractions represent average values in the region near the orifice of the hopper. It should also be emphasized that the designations of the flow types are based on visual observations of the flow, except for the data in the $2 \mathrm{D}$ hopper in which the pressure variations were also used to classify the data.

Figure 11 presents the nondimensional discharge rates for dry and lubricated flows (including all of the data shown in Fig. 6) as a function of $N$, the single-particle terminal velocity scaled by the characteristic hopper discharge velocity,

$$
N=w_{t} /\left[g\left(1-\rho_{f} / \rho_{s}\right) D^{\prime}\right]^{1 / 2} .
$$

For values of $N$ greater than 10 , the nondimensional discharge rates are close to unity. As $N$ drops below 10, which includes dry data, the discharge rates decrease. Note as shown in Fig. 9 for a large value of Re, the terminal velocity of a bed of material at $\phi=0.6$ is approximately one-tenth of the terminal velocity of a single particle. Hence, for $N<10$ the interstitial fluid affects the discharge rate because the particles cannot accelerate as they fall from the orifice.

\section{B. Pressure variations}

Although the discharge rates are steady, the corresponding measured total pressure varied during the measurement period. For some experiments, such as the dry and lubricated flows, it was possible to define an average value with variations about the mean. For other flows, such as channeling flows, the pressure varied continuously during the discharge period.

Initially the material was in the lower hopper and the transducers recorded the pressures for static conditions, as shown in Fig. 12 for $6 \mathrm{~mm}$ glass beads in air. The data acquisition was started before the hourglass was flipped and pressures were recorded until the upper hopper had emptied. In all of the dry flows, the fluid pressures were too small to be recorded by FP0, FP1, and FP2. The total pressures recorded by TP1 and TP2 showed large variations about a 


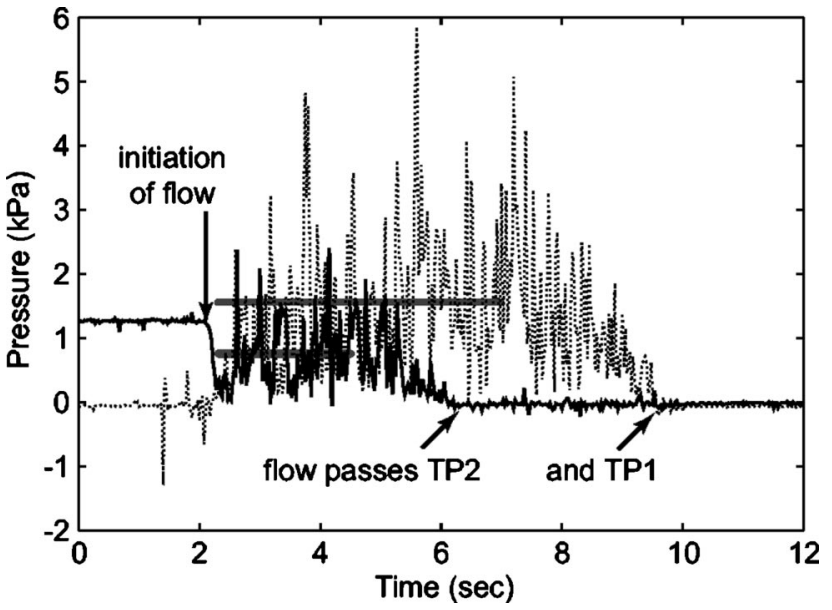

FIG. 12. The measured pressure as a function of time for TP1 and TP2 for $6 \mathrm{~mm}$ glass beads in air. The solid lines indicate the averaged values over a given time period. Initially the transducers are in the lower bin of the hourglass; hence TP2 gives an initial nonzero value of the pressure.

mean value with an initial and final transient period. The transient periods were neglected in calculating average value of the stresses. The average and standard deviations are given in Table I for both TP1 and TP2. The values of the TP2 during flow are smaller than under static conditions. This result may be due to changes during flow, but may also be due to the reduced height of material above the location of the pressure transducer, as explained below.

The analysis by Nedderman ${ }^{1}$ presents a balance of forces in an elemental section of a bunker of constant crosssectional area. Using $\sigma_{z z}$ as the mean stress along the axis of the bunker, and $z$ as the distance from the free surface into the bunker, the variation of $\sigma_{z z}$ in the $z$ direction is found from the difference between the specific weight of the material and the shear stress at the wall,

$$
\frac{D_{h}}{4} \frac{d \sigma_{z z}}{d z}=\tau_{w}-\frac{D_{h}}{4} \rho_{b} g
$$

The wall shear stress is equated to the friction factor and the averaged normal stress at the wall $\tau_{w}=\mu_{w} \sigma_{r r}$. An additional assumption is also made that the ratio of the stresses is a constant $K=\sigma_{r r} / \sigma_{z z}$. The value of $K$ depends on the type of

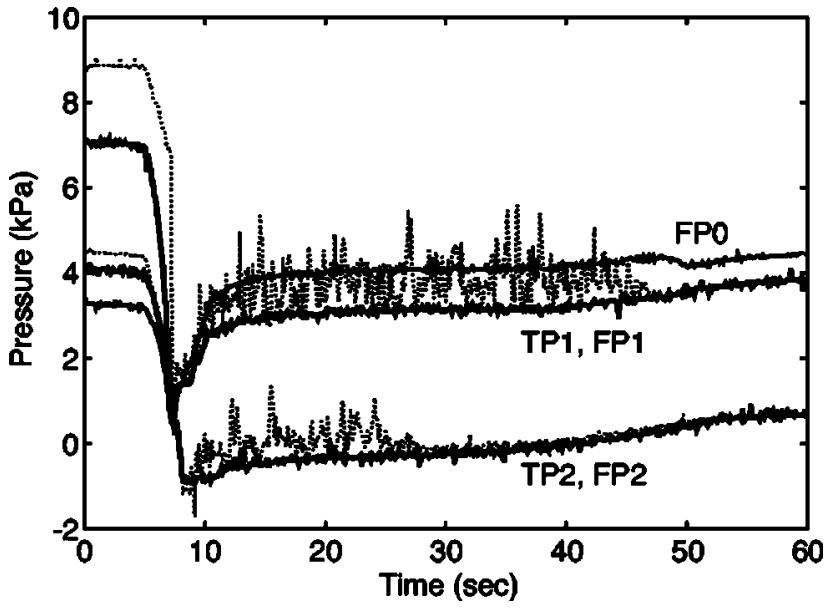

FIG. 13. The measured pressure (FP0, TP1, FP1, TP2, and FP2) as a function of time for $6 \mathrm{~mm}$ glass beads in water (lubricated). Initially the transducers are in the lower bin of the hourglass and particulate material covers both TP1 and TP2; the final pressure distributions are hydrostatic.

material and whether the material is in an active or passive state. Using the relation between the stresses, Eq. (5) can be integrated subject to the condition that the stress is zero at the free surface. When the material is in a passive state, the stress can reach its asymptotic value within $2 D_{h}$; in the active state the asymptotic value may be reached in $5 D_{h} .{ }^{1}$ For large values of $z$, the wall shear stress $\tau_{w}$ supports the weight of the particles. Using the asymptotic form of Eq. (5), the wall friction angle is written as

$$
\mu_{w}=D_{h} \rho_{b} g /\left(4 \sigma_{r r}\right) \text {. }
$$

For the 2D hourglass, the hydraulic diameter of the bin section is $6.8 \mathrm{~cm}$. Using the measured values of the normal stress (TP2) (where TP is total pressure) when the material is in the lower bin, the values for the friction factor were calculated using a stagnant solid fraction of 0.58 . The values of the friction factor range from 0.18 to 0.21 , and are listed along with TP2 in Table I for the different glass and steel particles.

In lubricated flows, the variations in the measured pressures are similar to those found for dry flows, but the magnitude of the fluctuations are reduced, as shown in Fig. 13 for

TABLE I. Friction factor and averaged stresses $(\mathrm{kPa})$ under stagnant and flowing conditions in the 2D hourglass.

\begin{tabular}{lcccc}
\hline \hline & $3 \mathrm{~mm}$ glass & $4 \mathrm{~mm}$ glass & $6 \mathrm{~mm}$ glass & $1.4 \mathrm{~mm}$ steel \\
\hline \multicolumn{1}{c}{ Dry } & & & & \\
Friction factor, $\mu_{w}$ & 0.18 & 0.18 & 0.19 & 0.21 \\
TP2, stagnant & 1.34 & 1.34 & 1.30 & 3.62 \\
TP2, discharge & $0.94 \pm 0.32$ & $0.90 \pm 0.50$ & $0.81 \pm 0.56$ & $1.76 \pm 1.25$ \\
TP1, discharge & $1.54 \pm 0.51$ & $1.41 \pm 0.72$ & $1.55 \pm 1.0$ & $3.28 \pm 0.50$ \\
$\quad$ & & & \\
$\quad$ With water & 0.16 & 0.15 & 0.15 & 0.19 \\
Friction factor, $\mu_{w}$ & 0.88 & 0.95 & 0.97 & 3.49 \\
EP2, stagnant & $0.29 \pm 0.16$ & $0.54 \pm 0.28$ & $0.47 \pm 0.24$ & $0.96 \pm 0.48$ \\
EP2, discharge & $0.65 \pm 0.21$ & $0.72 \pm 0.29$ & $0.78 \pm 0.50$ & $1.96 \pm 0.35$ \\
EP1, discharge & & & & \\
\hline \hline
\end{tabular}


$6 \mathrm{~mm}$ glass spheres in water. Once the particles pass the transducers, the total pressure remains at the value of the fluid pressure. At the orifice, the fluid pressure (FP0) remains near the hydrostatic level indicating that the hourglass walls, and not fluid drag, support the granular material.

Following the effective stress concept from soil mechanics ${ }^{10} \sigma_{\text {eff }}$ can also be defined from the difference between the total stress $\sigma_{t o t}$ and the fluid pressure $P_{f}$ :

$$
\sigma_{e f f}=\sigma_{t o t}-P_{f} \text {. }
$$

The values of the effective stress (EP1 and EP2) during flow and the value under static conditions (EP2) are given in Table I. The table also includes the friction factor using the definition given in Eq. (5). For this calculation the bulk density is taken as the relative density of the solid phase, $\rho_{b}$ $=\phi\left(\rho_{s}-\rho_{f}\right)$. Calculated in this way, the effective friction factor ranges from 0.15 to 0.19 , which is slightly smaller than found for dry flow. For all of the static experiments the values of the effective stress at location 2 were less than found under dry conditions. Similarly, when the material was flowing the effective stresses and the stress fluctuations at both locations were reduced as compared with the dry values. Part of the decrease may be due to the decrease in the relative bulk density; in addition, the stresses are affected by the changes in the flow structure.

During flow, the difference between the fluid pressures (such as FP0-FP1) is a result of the hydrostatic gradient, the pressure drop associated with flow within the packed bed, and the pressure drop associated with drag along the bounding walls. The latter term is often neglected in flows in porous media as compared with the particle drag; however, it may be significant in relatively porous beds. The pressure difference can be estimated from Ergun's equation for flow in a stationary porous medium, including inertial effects. ${ }^{1,11}$ Hence, the pressure gradient due to flow around the particles can be calculated as

$$
\frac{d P}{d z}=\frac{150 \phi^{2} \mu w_{f s}}{(1-\phi)^{3} d^{2}}+\frac{1.75 \phi \rho_{f} w_{f s}\left|w_{f s}\right|}{(1-\phi)^{3} d}
$$

where $\phi$ is the solid fraction, and $w_{f s}$ is the superficial velocity difference between particles and fluid $\left(w_{f s}=w_{f}-w_{s}\right)$, which is found from the bulk fluid volumetric flow rate in a stationary-particle frame of reference divided by the channel cross-sectional area. For an incompressible countercurrent flow, the superficial velocities of the interstitial fluid $w_{f}$ and speed of the solid particles $w_{s}$ are related through the following volumetric flow equation:

$$
\phi w_{s}=-(1-\phi) w_{f} .
$$

Since the flow is assumed to be one-dimensional, the superficial velocity difference is given by $w_{f s}=-w_{s} /(1-\phi)$. In a region of variable area $A(z)$, Ergun's equation can be integrated allowing for the fluid or solid velocity to vary with position $w_{s}(z)$ assuming an incompressible liquid,

$$
\left.w_{s} \phi A\right|_{z}=\left.w_{s} \phi A\right|_{z_{2}},
$$

where $z_{2}$ indicates the values of the velocity and area at measurement location 2 . Note that the calculation is greatly

\begin{tabular}{|c|c|c|c|c|}
\hline \multirow[b]{2}{*}{ Dia. (mm) } & \multirow{2}{*}{$\begin{array}{c}\text { Measured (kPa) } \\
\text { FP0-FP2 }\end{array}$} & \multicolumn{3}{|c|}{ Calculated (kPa) } \\
\hline & & 0.56 & 0.58 & 0.60 \\
\hline 3 & 2.1 & 1.8 & 2.3 & 2.6 \\
\hline 4 & 1.9 & 1.3 & 1.7 & 1.9 \\
\hline 6 & 1.6 & 1.3 & 1.6 & 1.8 \\
\hline
\end{tabular}

TABLE II. Comparison between measured pressure drops in water lubricated flow and pressure drop predictions using Ergun's equation using solid fractions of $0.56,0.58$, and 0.60 .

simplified if the solid fraction is assumed to be constant. For the measured mass flow rate, values of the calculated pressure difference from the orifice to position 2 are given in Table II for the experiments in the $2 \mathrm{D}$ hourglass using glass beads and water. The measured pressure differences are also given. In the calculations the solid fraction is assumed to be constant at $0.56,0.58$, and 0.60 . There is good correspondence between the measured and calculated pressure differences near $\phi=0.58$.

The pressure traces for the oscillatory flow are shown in Fig. 14 for $1.4 \mathrm{~mm}$ steel particle in water. Clearly, these traces differ from those for lubricated flows. The pressures measured at the orifice and at position 1 show an average decrease over time indicating that both the upward fluid flow and the walls support the mass of the particles. In addition, there are large-scale pressure fluctuations in the fluid due to the motion of the bubbles (see Fig. 3). At first the particles in the hopper are in a close-contact state and the bubble is at the lower edge of the orifice, FP0, FP1, and TP1 are at a local minimum and the interface location at the upper surface shows a slight decrease in solids flow rate. The rising bubble of fluid passes the pressure transducer, corresponding to an increase in fluid pressure and a break up of particles from the bridged state with a slight increase in solids flow rate. However, as the solids fraction increases, less fluid can pass in the opposite direction, resulting in a subsequent drop in fluid pressure and the beginning of the next cycle. Note that the

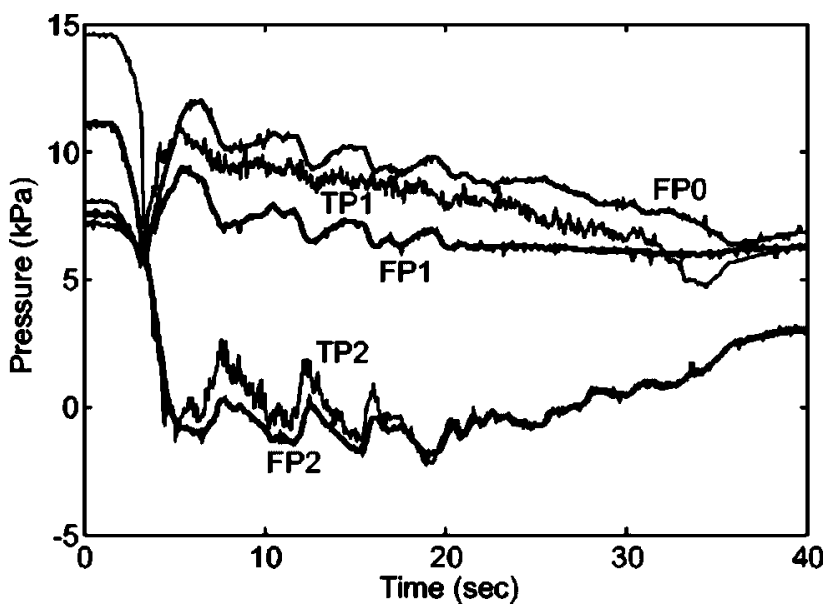

FIG. 14. The measured pressure (FP0, TP1, FP1, TP2, and FP2) as a function of time for $1.4 \mathrm{~mm}$ steel shot in water (oscillatory). Initially the transducers are in the lower bin of the hourglass and particulate material covers both TP1 and TP2; the final pressure distributions are hydrostatic. 


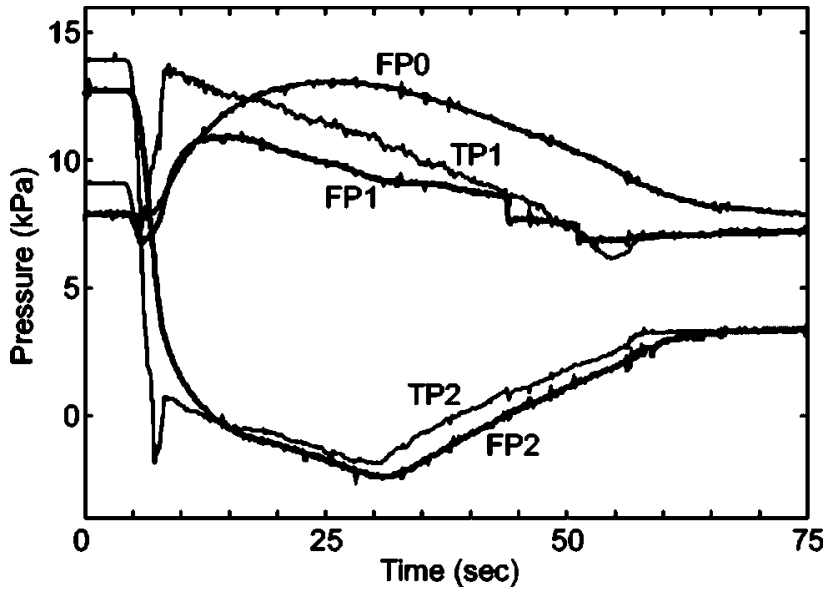

FIG. 15. The measured pressure (FP0, TP1, FP1, TP2, and FP2) as a function of time for $1.4 \mathrm{~mm}$ steel shot in $20 \mathrm{cP}$ water-glycerin mixture (fluidized). Initially the transducers are in the lower bin of the hourglass and particulate material covers both TP1 and TP2; the final pressure distributions are hydrostatic.

sensor at location 1 is within the fluidization region. The difference in the fluid pressures, FP0 and FP1 is $3 \mathrm{kPa}$, which is approximately equal to hydrostatic pressure of the suspended material, $\mathrm{FP} 0-\mathrm{FP} 1=\left[\phi \rho_{s}+(1-\phi) \rho_{f}\right] g\left(z_{0}-z_{1}\right)$, assuming that $\phi=0.5$. At position 2 , the fluid and total pressures show oscillations, but the average value does not change during the time when the material is above the sensor location. Hence, the material is expected to remain in a packed state in the upper region. Although the pressures varied over time, it was possible to calculate an effective stress at positions 1 and 2. These values are given in Table I, and the scaling of these stresses is similar to that found in the lubricated regime.

In fluidized flows, such as shown in Fig. 15 for the $1.4 \mathrm{~mm}$ steel shot in a water-glycerin mixture, the fluid and total pressure decreases with time at the orifice and at locations 1 and 2. The total pressure at position 1 (TP1) shows a linear decrease with time that follows the decrease in the height of the bed. After the initial transient, the fluid and total pressure at location 1 and 2 decrease with the height of granular material until the material passes the instrumentation location. However at location 1, the total pressure decreases at a rate that is faster than that of the fluid and so the effective stress decreases with the height of the bed. Hence, particles transmit some vertical stress to the sidewalls in this section of the hopper. As shown in Fig. 4, the upward fluid flow is concentrated in a jet near the orifice and cannot fully fluidize particles near the transducer that is located some distance from the orifice. At location 2, where upward fluid flow has become more uniformly distributed there is only a small difference between the fluid and total pressure traces indicating that the particles do not transmit much stress to the walls because they are almost fully fluidized.

In channeling flows, the total pressure tracks the fluid pressure, as shown in Fig. 16 for $3 \mathrm{~mm}$ glass spheres in a water-glycerin mixture. Except during the initial transient after the hourglass is inverted, the effective stress at both positions is negligible suggesting that the particles do not trans-

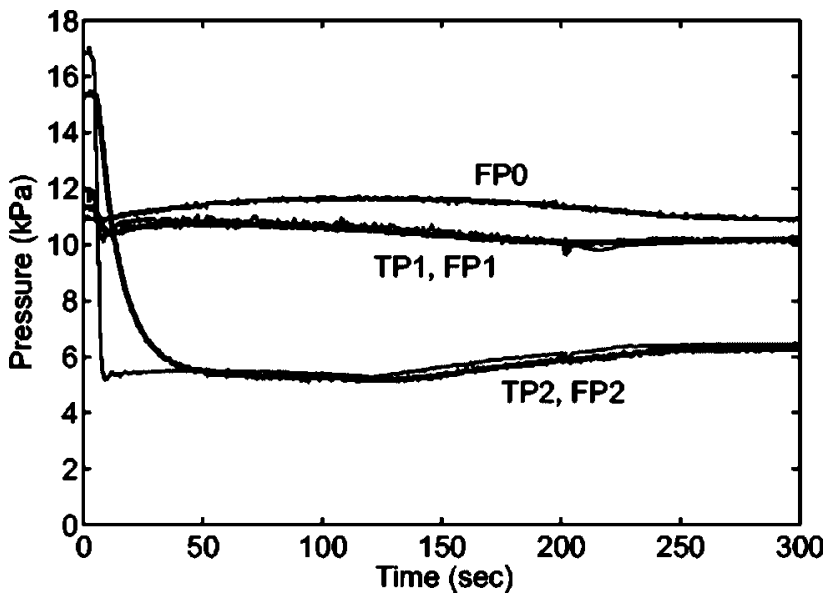

FIG. 16. The measured pressure (FP0, TP1, FP1, TP2, and FP2) as a function of time for $3 \mathrm{~mm}$ glass spheres in $96 \mathrm{cP}$ water-glycerin mixture (channeling). Initially the transducers are in the lower bin of the hourglass and particulate material covers both TP1 and TP2; the final pressure distributions are hydrostatic.

mit much stress to the walls. The fluid pressure measured at the orifice shows a slight increase and then a decrease over the discharge period as the weight of the material in the upper part of the hourglass decreases.

\section{Overall flow map}

Figure 17 presents a flow map in terms of the size ratio $d / D^{\prime}$ and the velocity ratio $N$ including flows and flows with an interstitial liquid for the $15^{\circ}$ and $30^{\circ}$ hourglasses, the data point corresponding to the world's largest hourglass, the data from the experiments by $\mathrm{Wu}$ et $a l^{2}$ and from Veje and Dimon. ${ }^{4}$ As shown in earlier sections, the data from the 2D and the $90^{\circ}$ hourglasses follow similar trends, but are not shown here because the geometries in the other studies correspond more closely with the $15^{\circ}$ and $30^{\circ}$ hourglasses.

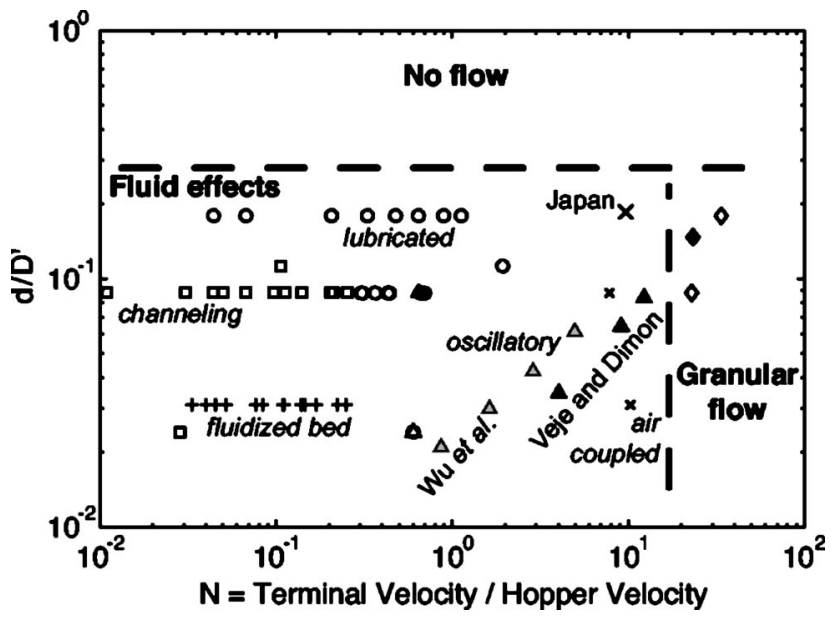

FIG. 17. Flow map of a discharging hourglass in terms of the ratio of the terminal velocity to the hopper discharge velocity as a function of $d / D^{\prime}$. The points include the data for the $15^{\circ}$ and $30^{\circ}$ geometries, as well as data corresponding to the world's largest hourglass (Japan), the ticking hourglass study by Wu et al. (gray filled), and from the study by Veje and Dimon (black filled). 
The data are divided into three main regions: no flow, steady granular flow without interstitial fluid effects, and flows in which the discharge rate is reduced because of interstitial fluid effects. The no-flow data, resulting from arching of particles across the orifice, occurred whenever the orifice opening was smaller than 5.5 particle diameters $\left(d / D^{\prime}>0.25\right.$ for the largest particles). Veje and Dimon ${ }^{4}$ observed that arching occurred for particles of $0.7 \mathrm{~mm}$ diameter, which corresponds with $d / D^{\prime}=0.32$ using $k=1.1$. Even with an interstitial liquid, arching occurred for the same values of $d / D^{\prime}$ as in a dry flow.

The granular flow region corresponds to flows in which the interstitial fluid does not affect the discharge rate, which includes data from this study as well as a single data point from the study by Veje and Dimon ${ }^{4}$ in which the flow maintained a constant discharge rate regardless of whether the upper hopper was open or closed to the atmosphere. The dividing line between the granular flow regime and the fluid influenced regime occurs at $N=10$ as described in the context of Fig. 11.

For the flows affected by the presence of the interstitial fluid, the map identifies the flow regime that was visually observed. For the data presented in this study, steady aircoupled flow occurred for $N \approx 10$; the flow became oscillatory at $N \approx 1$. The Japanese hourglass is viewed as a steady air coupled $\left(d / D^{\prime} \approx 0.2 ; N \approx 10\right)$ since the gas pressure within the hourglass controls the flow. For $1 \leqslant N \leqslant 10$, Wu et $a .^{2}$ and Veje and Dimon ${ }^{4}$ found unsteady air-coupled behavior. In this flow regime, surface roughness and shape of the particles affects the local permeability and unsteady flow characteristics of the medium, ${ }^{4}$ which results in some variability in the classification of the regimes.

The map also identifies the lubricated, channeling, and fluidized flows but does not contain firm boundaries because of the dependence on hourglass geometry. For $N<1$ and for the largest particle diameters (note the value of $D^{\prime}$ does not vary significantly in the experiments), the flows were lubricated. As the particle diameter decreased creating a less permeable bed, the flow formed channels along the sidewalls because the sidewall flow resistance decreased due to the lower voidage at the wall-particle interface as compared with the bulk of the bed. As the particle size was further decreased, the upward flow fluidized the bed. For values of $N<0.01$, the particles remained fluidized and did not form a well-defined bed.

\section{SUMMARY AND DISCUSSION}

This experimental investigation demonstrates that the flow in an hourglass or an enclosed vertical vessel can be divided into the following three regimes (i) granular flows with negligible interstitial fluid effects; (ii) flows affected by the presence of the interstitial fluid; and (iii) a no-flow region in which particles arch across the orifice and do not discharge. The flows in the fluid-affected region were visually classified as lubricated and air-coupled flows, oscillatory, channeling flows in which the flow preferentially rises along the sidewalls, and flows fluidized by countercurrent upward flow.
These flows are classified using the nondimensional parameter $N$, a ratio of the single-particle terminal velocity to the granular discharge velocity, and the ratio of the particle diameter to the effective length of the orifice $d / D^{\prime}$. When $N>10$ (a single particle falls rapidly through the fluid compared with the hopper discharge speed), the interstitial fluid effects are negligible and the discharge rate can be estimated using the characteristic hopper discharge speed. As the terminal velocity of the particle decreases (either by increasing the fluid viscosity or by decreasing the absolute magnitude of the density difference between the fluid and the particle), interstitial fluid effects increase and give rise to a variety of flow patterns that depend on flow geometry. In lubricated flows, where interstitial fluid effects are moderate, a modified Richardson-Zaki correlation can be used to find the reduction in discharge rate compared to when interstitial fluid effects are negligible assuming a solid fraction near 0.5. For fluidized and channeling flows, the modified Richardson-Zaki correlation is less appropriate; however, the discharge speeds can be estimated using a solid fraction near 0.3 .

Besides the visual observations of the discharge patterns, the pressure measurements show that in the lubricated flow regime, Ergun's equation gives an adequate description of the pressure variation through the granular material. Using the concept of an effective stress for lubricated flows, a friction factor can be defined that is slightly lower $\left(\mu_{w} \approx 0.16\right)$ than found in a dry granular medium $\left(\mu_{w} \approx 0.19\right)$. Hence, in a lubricated flow the weight of the particles is supported by the sidewalls. For the fluidized and channeling flows, the sidewalls do not support the weight of the bed; as a result the fluid and effective wall stresses continually vary as the materials are discharged.

It should be noted that Ergun's equation and the drag correlations used are experimental relations and not rigorous theoretical derivations. It is to be expected for the predictions based on those models to only be accurate for conditions similar to those for which the relations were obtained. Further modeling of solid-liquid flows is currently underway.

\section{ACKNOWLEDGMENTS}

This work was supported by the National Science Foundation Grant Nos. CTS-9908430 and CTS-0314005 and Caltech's Summer Undergraduate Research Fellowship. The authors thank Professor Ronald F. Scott for his helpful suggestions.

${ }^{1}$ R. M. Nedderman, Statics and Kinematics of Granular Materials (Cambridge University Press, Cambridge 1992).

${ }^{2}$ X. Wu, K. J. Måløy, A. Hansen, M. Ammi, and D. Bideau, "Why hour glasses tick,” Phys. Rev. Lett. 71, 1363 (1993).

${ }^{3}$ T. Le Pennec, K. J. Måløy, A. Hansen, M. Ammi, D. Bideau, and X. Wu, "Ticking hour glasses: Experimental analysis of intermittent flow," Phys. Rev. E 53, 2257 (1996).

${ }^{4}$ C. T. Veje and P. Dimon, "The dynamics of granular flow in an hourglass," Granular Matter 3, 151 (2001).

${ }^{5}$ J. R. Ducker, M. E. Ducker, and R. M. Nedderman, "The discharge of granular materials from unventilated hoppers," Powder Technol. 42, 3 (1985).

${ }^{6}$ R. Clift, J. R. Grace, and M. E. Weber, Bubbles, Drops and Particles (Academic, New York, 1978).

${ }^{7}$ E. S.G. Shaqfeh and A. Acrivos, "Enhanced sedimentation in vessels with inclined walls: Experimental observations," Phys. Fluids 30, 1905 (1987). 
${ }^{8}$ J. F. Richardson and W. N. Zaki, "Sedimentation and fluidisation: Part I," Trans. Inst. Chem. Eng. 32, S82 (1954).

${ }^{9} \mathrm{R}$. DiFelice, "The voidage function for fluid-particle interaction systems," Int. J. Multiphase Flow 20, 153 (1994).
${ }^{10}$ R. F. Scott, Principles of Soil Mechanics (Addison-Wesley, New York, 1963).

${ }^{11}$ S. Ergun, "Fluid flow through packed columns," Chem. Eng. Prog. 48, 89 (1952). 